\title{
Emerging Clinical Imaging Techniques for Spinal Arteriovenous Malformations
}

\author{
Peter G. Campbell, MD', Lisa Tartaglino, MD², Hayan Dayoub, MD', \\ Pascal M. Jabbour, MD', Aaron S. Dumont, MD', L. Fernando Gonzalez, MD', \\ Robert H. Rosenwasser', MD, Stavropoula I. Tjoumakaris, MD' \\ ${ }^{1}$ Department of Neurological Surgery, Thomas Jefferson University, Philadelphia, PA \\ 2Jefferson Hospital for Neuroscience, Philadelphia, PA
}

Key Words: MRI, spine, spinal, arteriovenous malformation, dural arteriorvenous fistula, AVF

Running Title: Clinical Imaging for Spinal dAVF

\section{Introduction}

Spinal arteriovenous malformations (SAVMs) are rare and under-diagnosed entities. If untreated, SAVMs can lead to progressive spinal cord myelopathy. The diversion of arterial blood through dorsal and/or medullary veins can lead to a vascular steal phenomena often accompanying highflow lesions, or venous hypertension and congestion which ultimately reduces intramedullary blood flow in lower flow malformations ${ }^{1}$. Therefore, timely diagnosis and a precise understanding of these lesions can determine surgical strategies and prevent delays in treatment.

The "gold standard" diagnostic study to diagnose and evaluate these entities has traditionally been catheter-based intra-arterial digital subtraction angiography (DSA) secondary to its ability to render images with temporal and spatial resolution ${ }^{2}$. However, even routine spinal angiography has been associated with spinal cord infarct and renal failure ${ }^{3,4}$. In the setting of a SAVM, selective catheterization of arteries supplying a SAVM can often be time consuming, require multiple catheterizations, involve long radiation exposure times, and use large volumes of potentially nephrotoxic contrast agents $^{1}$. Furthermore, selective catheterization of segmental arteries can lead to spinal cord infarction due to the catheter-related vasospasm. The purpose of this report is to review recent advancements in the magnetic resonance imaging (MRI) of SAVMs that could lead to more timely diagnoses while reducing the complication profile of spinal angiography.

\section{Diagnostic Imaging of SAVMs}

Initially, noninvasive spine imaging is typically performed in the setting of a suspected SAVM. MRI is often carried out to evaluate the spinal column, cord, vasculature and supporting structures. SAVM are often suspected by the demonstration of prominent or conspicuous subarachnoid vessels on with traditional MR imaging techniques. Unfortunately, these standard MR imaging characteristics are nonspecific and lack temporal resolution. This imaging evaluation does not provide for localization of the fistulous connections of the SAVM, which consequently limits its clinical utility in planning definitive treatment plans.

However, a new imaging technique called timeresolved imaging of contrast kinetics (TRICKS) is a new technique in 3D MR angiography that can

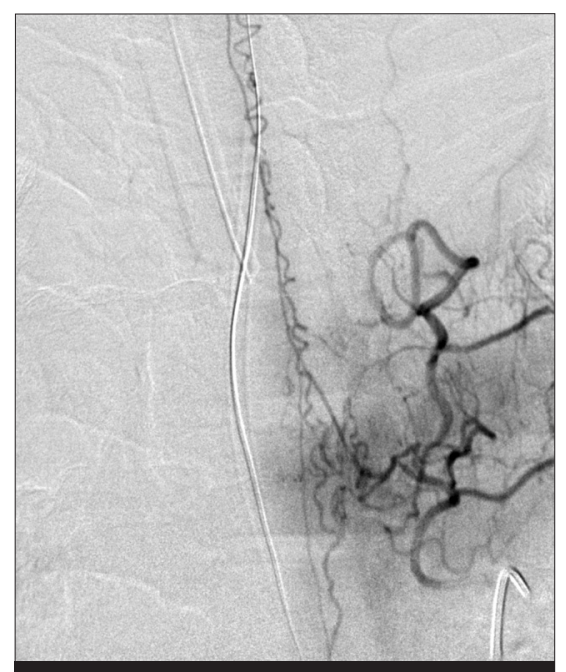

Figure 1

Initial spinal angiography of Left T4 spinal segmental artery, showing a Type I arteriovenous fistula with ascending and descending dilated venous drainage. achieve an adequate temporal and spatial resolution to evaluate AVMs especially when combined with 3 Tesla imaging hardware. ${ }^{5-7}$ The TRICKS imaging protocol has surmounted the limitations of conventional MR imaging upon temporal resolution, by providing images with a temporal resolution of 3-6 seconds and a spatial resolution of approximately $1 \mathrm{~mm}^{1}$. This allows for the AV shunt zone to be visualized, the predominant arterial feeders can be localized, and the venous drainage pattern to be displayed in a single MRA examination. ${ }^{8}$

\section{Utilization of TRICKS in Recurrent} Spinal Arteriovenous Malformations Conventional spinal angiography for the diagnosis of recurrent or residual spinal arteriovenous malformations continues to be a challenge for neuro-interventionalists. Previously clipped or embolized spinal arterial pedicles can be extremely challenging to identify and catheter-

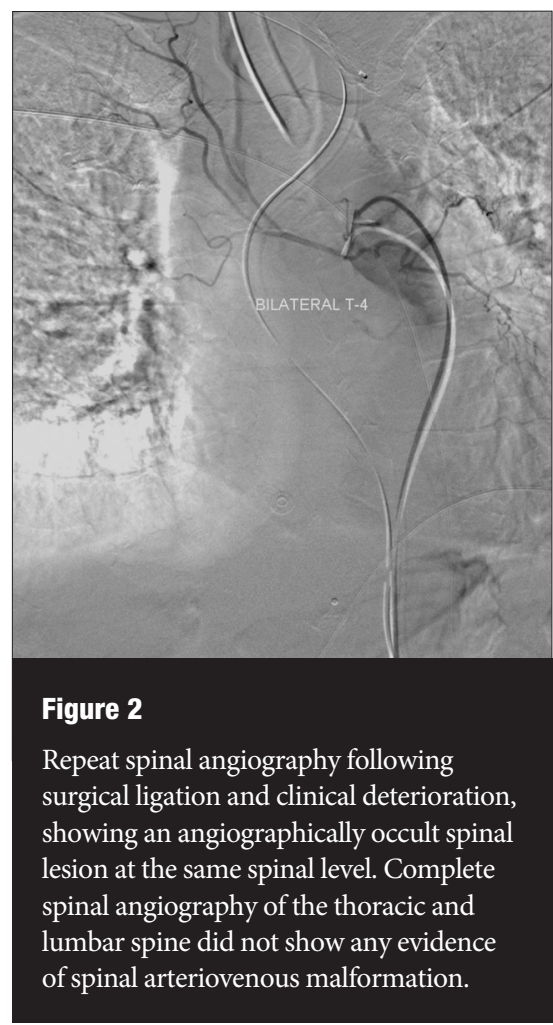



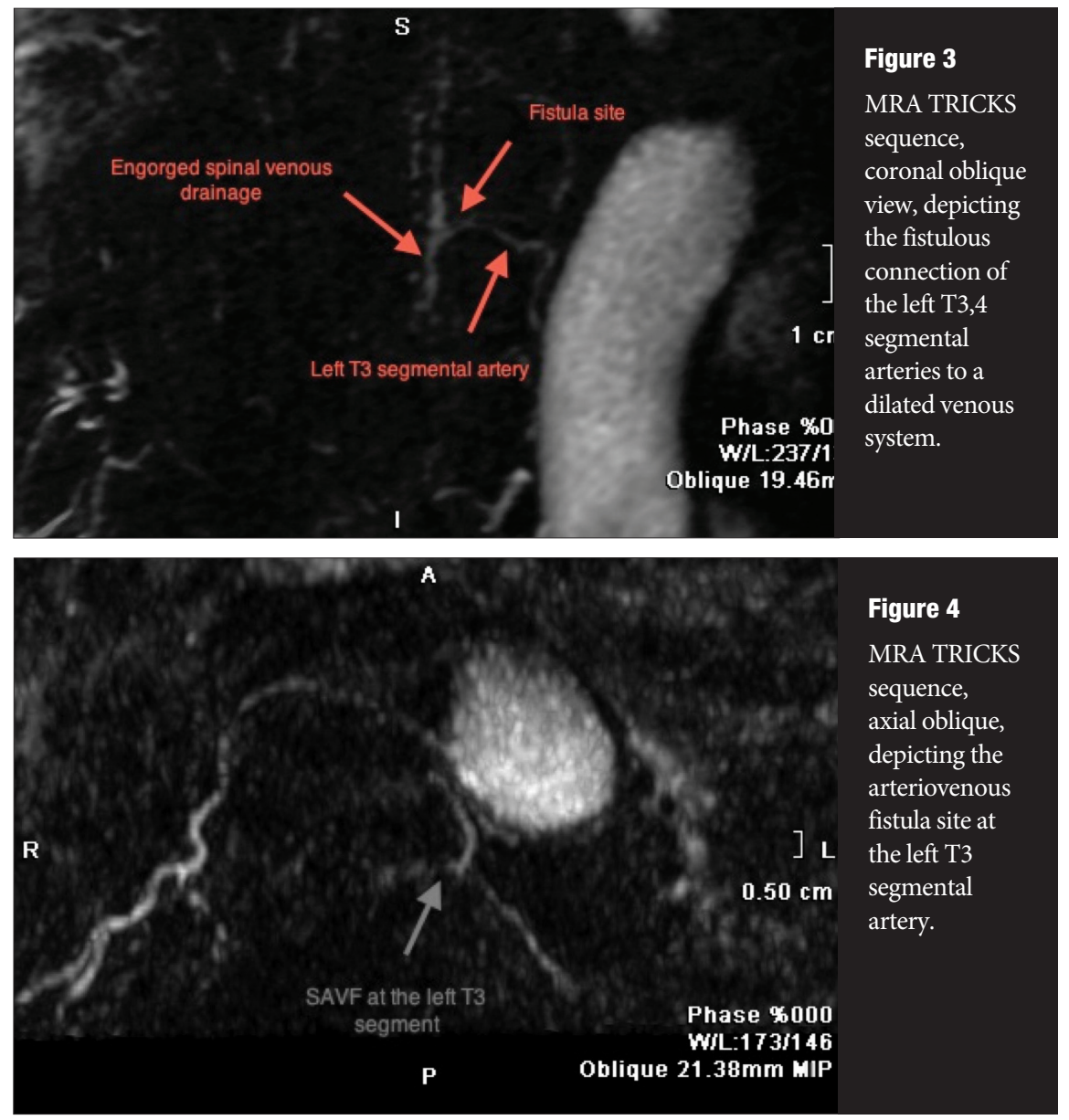

ize during spinal angiography. Furthermore, these patients have higher complication rates from spinal angiography due to their already compromised spinal cord.

In our institution, the utilization of TRICKS MRA in such patients has been extremely useful in the diagnosis of recurrent SAVM. Several angiographically occult spinal lesions have been identified and treated with the aid of this study. As a result, TRICKS MRA is emerging as an adjunct diagnostic tool in the neuro-interventionalist's armamentarium for the management of SAVM.

Figures 1-4 depict the radiographic progression of aSAVM. Figure 1 shows the spinal angiogram of left T3-4 SAVM, type I spinal arteriovenous fistula. Following surgical ligation of the fistula, clinical deterioration of the patient led to a second spinal angiogram, which was negative (Figure 2). An MRA TRICKS sequence was performed (Figures 3,4) which showed a small recurrent SAVF at the same level.

\section{Conclusion}

Continuing improvement of noninvasive imaging MRA protocols will provide neurosurgeons with more precise localization of SAVMs. This development has the potential to minimize the procedural time needed for spinal DSA and the complication profile for patients undergoing follow-up imaging. Additionally, this technique will provide valuable clinical information for treatment decisions regarding endovascular therapy, open surgical treatment in addition to a potential to decrease the procedural complication profile for patients undergoing follow-up imaging.

\section{References}

1. Eddleman CS, Jeong H, Cashen TA, et al. Advanced noninvasive imaging of spinal vascular malformations. Neurosurg Focus 2009;26:E9.

2. Krings T, Lasjaunias PL, Hans FJ, et al. Imaging in spinal vascular disease. Neuroimaging Clin N Am 2007;17:57-72.

3. Kieffer E, Fukui S, Chiras J, Koskas F, Bahnini A, Cormier E. Spinal cord arteriography: a safe adjunct before descending thoracic or thoracoabdominal aortic aneurysmectomy. J Vasc Surg 2002;35:262-8.
4. Luetmer PH, Lane JI, Gilbertson JR, Bernstein MA, Huston J, 3rd, Atkinson JL. Preangiographic evaluation of spinal dural arteriovenous fistulas with elliptic centric contrast-enhanced MR Angiography and effect on radiation dose and volume of iodinated contrast material. AJNR Am J Neuroradiol 2005;26:711-8.

5. Chen Q, Quijano CV, Mai VM, et al. On improving temporal and spatial resolution of 3D contrast-enhanced body MR angiography with parallel imaging. Radiology 2004;231:893-9.

6. Gauvrit JY, Leclerc X, Oppenheim C, et al. Three-dimensional dynamic MR digital subtraction angiography using sensitivity encoding for the evaluation of intracranial arteriovenous malformations: a preliminary study. AJNR Am J Neuroradiol 2005;26:1525-31.

7. Korosec FR, Frayne R, Grist TM, Mistretta CA. Timeresolved contrast-enhanced 3D MR angiography. Magn Reson Med 1996;36:345-51.

8. Mull M, Nijenhuis RJ, Backes WH, Krings T, Wilmink JT, Thron A. Value and limitations of contrast-enhanced MR angiography in spinal arteriovenous malformations and dural arteriovenous fistulas. AJNR Am J Neuroradiol 2007;28:1249-58.

\section{Corresponding Author}

Stavropoula I. Tjoumakaris, MD

Department of Neurological Surgery

Thomas Jefferson University

909 Walnut St, 2nd Floor

Philadelphia, PA 19107

Email: stavropoula.tjoumakaris@jefferson.edu Phone: 215-503-7008 